\title{
Blood Concentrations of 1,4-Dioxane in Humans after Oral Administration Extrapolated from In Vivo Rat Pharmacokinetics, In Vitro Human Metabolism, and Physiologically Based Pharmacokinetic Modeling
}

\author{
Ryohji Takano, ${ }^{a, b}$ Norie Murayama, ${ }^{a}$ Kana Horiuchi, ${ }^{a}$ Masato Kitajima, ${ }^{b}$ \\ Fumiaki Shono, ${ }^{c}$ and Hiroshi Yamazaki ${ }^{*, a}, d$ \\ ${ }^{a}$ Laboratory of Drug Metabolism and Pharmacokinetics, Showa Pharmaceutical University, 3-3165 Higashi-tamagawa Gakuen, \\ Machida, Tokyo 194-8543, ${ }^{b}$ Fujitsu Kyusyu Systems, Momochihama, Sawara-ku, Fukuoka 814-8589, 'Japan Chemical Industry As- \\ sociation (JCIA), 1-4-1 Shinkawa, Chuo-ku, Tokyo 104-0033, Japan and ${ }^{d}$ High Technology Research Center, Showa Pharmaceutical \\ University, 3-3165 Higashi-tamagawa, Gakuen, Machida, Tokyo 194-8543, Japan
}

(Received May 8, 2010; Accepted June 2, 2010; Published online June 9, 2010)

\begin{abstract}
The present study defined a simplified physiologically based pharmacokinetic (PBPK) model for 1,4-dioxane in humans based on in vitro metabolic parameters determined using relevant liver microsomes, coefficients derived in silico, physiological parameters derived from the literature, and a developed PBPK model in rats. The model consists of a chemical absorption compartment, a metabolizing compartment, and a central compartment for 1,4dioxane. Evaluation of the rat model was performed by comparisons with experimental pharmacokinetic values from blood and urine obtained from rats in vivo after daily oral treatment with 1,4-dioxane $(500 \mathrm{mg} / \mathrm{kg}$, a noobserved-adverse-effect level) for 14 days. Elimination rates of 1,4-dioxane in vitro were established using data from rat liver microsomes and from pooled human liver microsomes. 1,4-Dioxane was expected to be absorbed and cleared rapidly from the body in silico, as was the case for rats confirmed experimentally in vivo with repeated low-dose treatments. These results indicate that the simplified PBPK model for 1,4-dioxane is useful for a forward dosimetry approach in humans. This model may also be useful for simulating blood concentrations of other related compounds resulting from exposure to low chemical doses.
\end{abstract}

Key words — physiologically based biokinetic modeling, cytochrome P450, simulation, no-observed-adverseeffect level, biomonitoring, human liver microsomes

\section{INTRODUCTION}

Basic information is necessary to interpret human biomonitoring results obtained internationally to promote risk-based decision making. ${ }^{1-3)}$ Simplified, advanced, and accurate risk assessment systems are of global interest to support appropriate interpretation and communication based on human biomonitoring results. ${ }^{4)}$ Pharmacokinetic and/or toxicokinetic parameters for a variety of chemicals have been determined in animal toxi-

\footnotetext{
${ }^{*}$ To whom correspondence should be addressed: Laboratory of Drug Metabolism and Pharmacokinetics, Showa Pharmaceutical University, 3-3165 Higashi-tamagawa Gakuen, Machida, Tokyo 194-8543, Japan. Tel. \& Fax: +81-42-721-1406; Email: hyamazak@ac.shoyaku.ac.jp
}

cology studies, even when limited corresponding data exist for humans. ${ }^{5)}$ Species differences of drugmetabolizing enzymes in the liver, including cytochrome P450 (P450 or CYP) enzymes, are the focus for understanding qualitative and quantitative differences in concentrations in biological fluids or chemical exposures in animals and in humans. ${ }^{6}$ ) It has been generally attempted to collect extensive information regarding specific physiologically based pharmacokinetic (PBPK) models found in the literature for predicting concentrations in various biological fluids following multiple dose exposures. ${ }^{2)}$ However, although simple, inexpensive, and reliable methods are needed for evaluating the accurate toxic risk, only very complicated models have so far been established. ${ }^{7,8)}$ 
1,4-Dioxane (Fig. 1) is widely used, primarily as a solvent or as a solvent stabilizer, ${ }^{9)}$ but subchronic oral toxicity of 1,4-dioxane has been examined in rats by administering 1,4-dioxane in drinking water, suggesting hepatic and renal failures. ${ }^{10}$ ) 1,4-Dioxane is believed to be primarily metabolized by P450s to 2-(2-hydroxyethoxy)acetic acid or its interconversion product 1,4-dioxiane-2-one (Fig. 1). ${ }^{7,8)}$ Kinetic models for 1,4-dioxane and its major metabolite (hydroxyethoxyacetic acid) were published in $\left.1990 ;{ }^{11}{ }^{12}\right)$ however, the uncertainties and deficiencies of these models for use in a contemporary cancer risk assessment for 1,4-dioxane have been pointed out. ${ }^{8)}$

Therefore, the purpose of the present study was to carry out a forward dosimetry approach (shown in Fig. 2) using data from chemical doses administered to animals and from in vitro experiments with

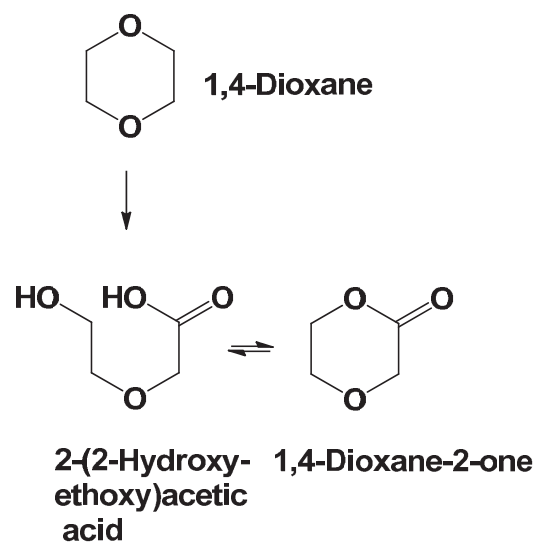

Fig. 1. Chemical Structures of 1,4-dioxane and Reported Metabolites liver microsomes from humans and animals to predict 1,4-dioxane concentrations in humans. We report herein that the adjusted animal biomonitoring equivalents after orally administered doses at a noobserved-adverse-effect level (NOAEL) in rat studies were scaled to human biomonitoring equivalents using known species allometric scaling factors and human metabolic data with a simple PBPK model.

\section{MATERIALS AND METHODS}

Chemicals, Animals, and Enzyme Preparations - 1,4-Dioxane was obtained from Wako Pure Chemicals (Osaka, Japan). Male SpragueDawley rats (7 weeks old, $180 \mathrm{~g}$ ) were treated daily with 1,4 -dioxane $[500 \mathrm{mg} / \mathrm{kg}$ body weight (bw)] per os (p.o.) for 14 days, based on a NOAEL dose. ${ }^{8)}$ This study was approved by the experimental animal committee of Showa Pharmaceutical University. In separated experiments, liver microsomes from male Sprague-Dawley rats (7 weeks old, $180 \mathrm{~g}$ ) intraperitoneal (i.p). treated with 1,4dioxane $(500 \mathrm{mg} / \mathrm{kg})$ according to typical P450 induction methods and from untreated controls were prepared as described previously. ${ }^{13)}$ Microsomal P450 contents were determined spectrally by the established method. ${ }^{14)}$ Protein concentrations were estimated by using a bicinchoninic acid (BCA) protein assay kit (Pierce, Rockford, IL, U.S.A.). Pooled liver microsomes from humans (H150) were obtained from BD Biosciences (Woburn, MA, U.S.A.); the human subjects might not be exposed by 1,4-dioxane. Typical P450 substrates, their reac-

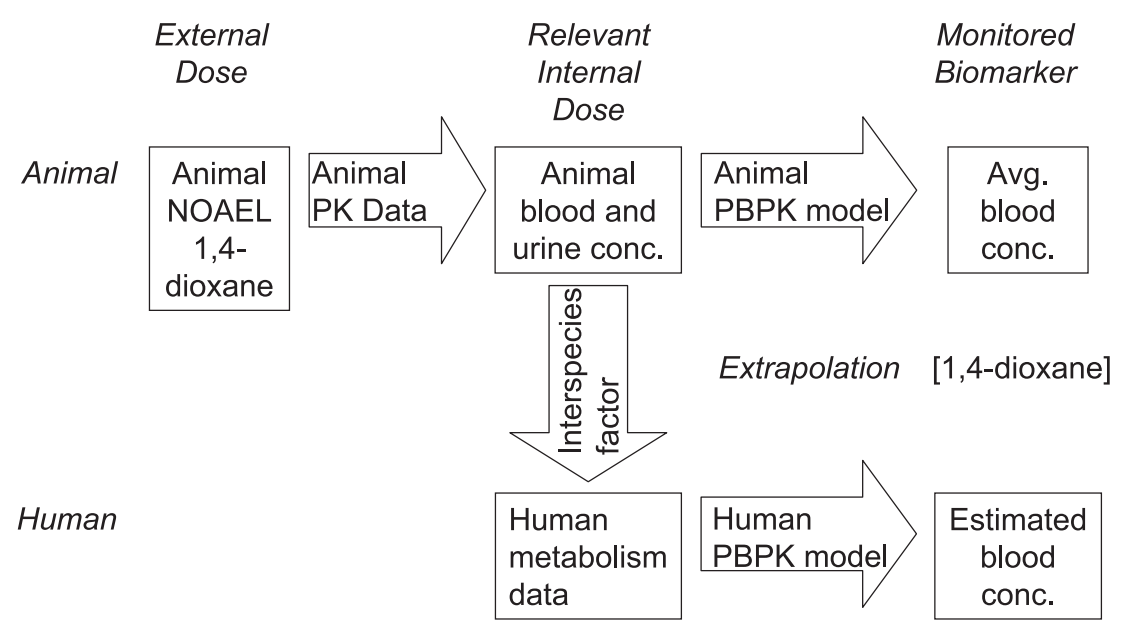

Fig. 2. Approach for Calculating Blood-based Biomonitoring Equivalents for 1,4-dioxane PK, pharmacokinetics. 
Table 1. Parameters Used for the rat PBPK Model

\begin{tabular}{lccc}
\hline \hline Parameter & Symbol & 1,4 -Dioxane & Unit \\
\hline Octanol-water partition coefficient & $\log P$ & -0.31 & \\
Hepatic intrinsic clearance & $C L_{h, \text { int }}$ & 0.0244 & $1 / \mathrm{h}$ \\
Liver-plasma concentration ratio & $K_{p, h}$ & 0.692 & - \\
Renal clearance & $C L_{r}$ & 0.000290 & $1 / \mathrm{h}$ \\
Plasma unbound fraction & $f_{u, p}$ & 0.806 & - \\
Ratio of the blood to plasma concentration & $R_{b}$ & 1.00 & - \\
Volume of systemic circulation & $V_{1}$ & 0.0810 & 1 \\
Hepatic volume & $V_{h}$ & 0.00850 & 1 \\
Hepatic blood flow rate of systemic circulation to the tissue compartment & $Q_{h}$ & 0.853 & $1 / \mathrm{h}$ \\
Absorption rate constant & $k_{a}$ & 0.280 & $\mathrm{~h}^{-1}$ \\
Fraction absorbed $\times$ intestinal availability & $F_{a} F_{g}$ & 1.00 & - \\
Dose & Dose & 125 & $\mathrm{mg}$ \\
\hline
\end{tabular}

tion products, and other reagents used in this study were obtained from sources described previously or were of the highest quality commercially available. $^{13,15)}$

Typical P450-dependent marker oxidation activities were measured in liver microsomes in rats to evaluate enzyme inductions on treatment with 1,4-dioxane. Activities for the $O$-dealkylation of ethoxyresorufin $(20 \mu \mathrm{M}$, for CYP1A) and pentoxyresorufin $(100 \mu \mathrm{M}, \mathrm{CYP} 2 \mathrm{~B})$ and for testosterone $7 \alpha$-hydroxylation $(200 \mu \mathrm{M}, \mathrm{CYP} 2 \mathrm{~A})$, tolbutamide methyl hydroxylation $(1000 \mu \mathrm{M}, \mathrm{CYP} 2 \mathrm{C})$, bufuralol 1'-hydroxylation (20 $\mu \mathrm{M}, \mathrm{CYP} 2 \mathrm{D})$, chlorzoxazone 6-hydroxylation $(50 \mu \mathrm{M}, \mathrm{CYP} 2 \mathrm{E})$, and midazolam $1^{\prime}-$ and 4-hydroxylation $(100 \mu \mathrm{M}$, CYP3A) were assayed according to the described HPLC methods. ${ }^{13,16,17)}$

\section{1,4-Dioxane Determinations in Biological Sam-} ples from Rats — Plasma and urine samples from individual rats were diluted 10 times with water. 1,4-Dioxane concentrations in these samples were measured by a gas chromatograph/mass spectrometer (GC/MS) with a head-space sampler system as reported previously (GCMS-QP2010, Shimadzu, Kyoto, Japan $)^{8)}$ equipped with a CPPORABOND Q column $(25 \mathrm{~m} \times 0.24 \mathrm{~mm} \times 3 \mu \mathrm{m}$, Chrompack). To determine the concentrations, the intensities of the precursor ions for 1,4-dioxane $(m / z=88)$ and internal standard 1,4-dioxane- $\mathrm{d}_{3}$ $(m / z=96)$ were used.

Human Metabolic Study — Elimination rates of 1,4-dioxane in liver microsomes from humans and rats were measured by the GC/MS system described above and were compared. Briefly, a typical incubation mixture consisted of $100 \mathrm{mM}$ potassium phosphate buffer ( $\mathrm{pH} 7.4$ ), an NADPH-generating system, a substrate $(50 \mu \mathrm{M})$, and liver microsomes $(0.50 \mathrm{mg}$ protein $/ \mathrm{ml})$ in a final volume of $0.25 \mathrm{ml}$. Incubations were carried out at $37^{\circ} \mathrm{C}$ for $30 \mathrm{~min}$. The assay linearity with respect to time and protein concentration and the reproducibility (within $<15 \%$ ) were confirmed. The incubation was terminated by adding $0.40 \mathrm{ml}$ of ice-cold acetonitrile.

Estimation of 1,4-dioxane Concentrations by PBPK Modeling with Suitable Parameters

A simplified PBPK model was set up as described previously. ${ }^{8,18)}$ Parameter values for the physicochemical properties of compounds $\left(f_{u, p}, \log P, K_{p, h}\right.$, and $R_{b}$ ) are shown in Table 1 . Values of $f_{u, p}$ and $\log P$ were obtained by in silico estimation using SimCYP and ChemDrawBioUltra software, ${ }^{19)} K_{p, h}$ was estimated from these two values (Appendix A), and $R_{b}$ was assumed to be 1.0 (blood and plasma concentrations are assumed to be equal). Parameter values which represent physiological properties such as hepatic volumes and blood flow rates in rats or humans were taken from the literature. ${ }^{8,18)}$

Experimental plasma concentrations of 1,4dioxane were analyzed by WinNonlin software (Professional version 5.01) with a one-compartment model and yielded primary $k_{a}$, elimination constant $\left(k_{e l}\right)$. Values of total clearance $\left(C L_{\mathrm{tot}}\right)$, hepatic clearance $\left(C L_{h}\right), C L_{h, \text { int }}$, and $V_{1}$ were also calculated (Appendix B). Subsequently, final parameter values for the rat PBPK model were calculated using the initial values mentioned above by the user model in WinNonlin and are shown in Table 1. Consequently, the following systems of differential equations were solved to conduct the modeling shown in Fig. 3:

$$
\frac{d X_{g}(t)}{d t}=-k_{a} \times X_{g}(t) \quad \text { where at } t=0,
$$




$$
\begin{aligned}
X_{g}(0)= & F_{a} \times F_{g} \times \text { Dose }, \\
V_{h} \frac{d C_{h}}{d t}= & Q_{h} \times C_{b}-\frac{Q_{h} \times C_{h} \cdot R_{b}}{K_{p, h}}+k_{a} \\
& \times X_{g}-C L_{h, \text { int }} \times \frac{C_{h}}{K_{p, h}} \times f_{u, p}, \\
V_{1} \frac{d C_{b}}{d t}= & -Q_{h} \times C_{b}+\frac{Q_{h} \times C_{h} \times R_{b}}{K_{p, h}}-C L_{r} \times C_{b},
\end{aligned}
$$

where $X_{g}$ is the substance amount in the gut, $C_{h}$ is the hepatic substance concentration, and $C_{b}$ is the blood substance concentration.

To define a simplified PBPK model for 1,4dioxane in humans based on the rat PBPK model, we used relevant liver microsomes and physiological parameters $\left(C L_{r}, k_{a}\right.$, and $\left.V_{1}\right)$ derived from the literature and applied the systems approach to fit them into the traditional parallelogram for risk assessment, ${ }^{2)}$ as shown in Fig. 2 (Appendix C). The in vivo $C L_{h, \text { int }}$ of 1,4-dioxane in humans was estimated by multiplying the calculated initial parameter value for in vitro $C L_{h \text {,int }}$ in humans by the ratio of in vivo to in vitro $C L_{h, \text { int }}$ values in rats, as mentioned above for modeling in rats. Then, the final parameters for the PBPK model in humans were calculated

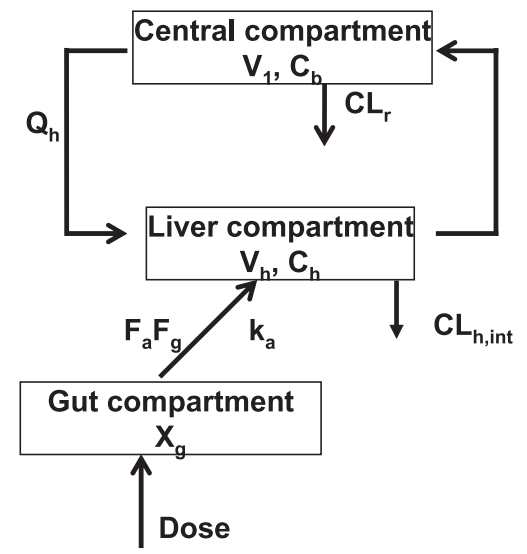

Fig. 3. PBPK Model Established in this Study for Rats and Humans using these initial values by the methods in Appendix $\mathrm{C}$ and are shown in Table 2. As was done for the rat model, systems of differential equations were solved to obtain concentrations in each model compartment in humans.

\section{RESULTS}

\section{PBPK Model for Rats Orally Treated with 1,4- dioxane}

To obtain detailed kinetic parameters, male rats were orally treated with 1,4-dioxane according to the protocol for general repeated exposure tests. Figure 3 shows the mean levels of 1,4-dioxane in blood and urine from rats after the final treatment of 14 daily repeated doses of 1,4-dioxane $(500 \mathrm{mg} / \mathrm{kg})$. 1,4-Dioxane was rapidly absorbed and immediately cleared within a day (Fig. 4A). Urinary excretion of 1,4-dioxane was almost complete within $24 \mathrm{hr}$ after the final repeated administration (Fig. 4B). Renal clearance $\left(C L_{r}\right)$ values of 1,4-dioxane were calculated from the amount excreted into the urine $(2.25 \mathrm{mg})$ divided by the area under the blood curve
(A)

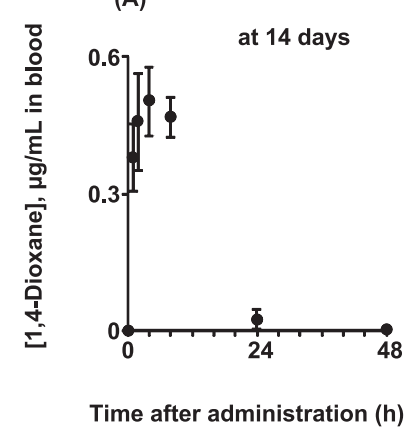

(B)

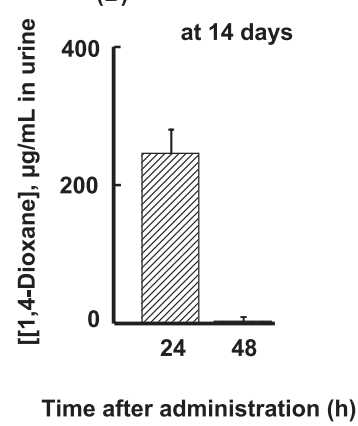

Fig. 4. PK Profiles in Rats p.o. Treated with 1,4-dioxane 1,4-Dioxane concentrations in blood (A) and urine (B) were determined in rats treated with 1,4-dioxane $(500 \mathrm{mg} / \mathrm{kg}$ per day) after the final administration of 14 daily doses.

Table 2. Parameters Used for the Human PBPK Model

\begin{tabular}{lccc}
\hline \hline Parameter & Symbol & $1,4-$ Dioxane & Unit \\
\hline Hepatic intrinsic clearance & $C L_{h, \text { int }}$ & 1.76 & $1 / \mathrm{h}$ \\
Renal clearance & $C L_{r}$ & 0.0873 & $1 / \mathrm{h}$ \\
Volume of systemic circulation & $V_{1}$ & 23.7 & 1 \\
Hepatic volume & $V_{h}$ & 1.50 & 1 \\
Hepatic blood flow rate of systemic circulation to the tissue compartment & $Q_{h}$ & 96.6 & $1 / \mathrm{h}$ \\
Absorption rate constant & $k_{a}$ & 0.208 & $\mathrm{~h}^{-1}$ \\
Dose & Dose & 35000 & $\mathrm{mg}$ \\
\hline
\end{tabular}

Other parameters are the same as those shown in Table 1 for the rat PBPK model. 


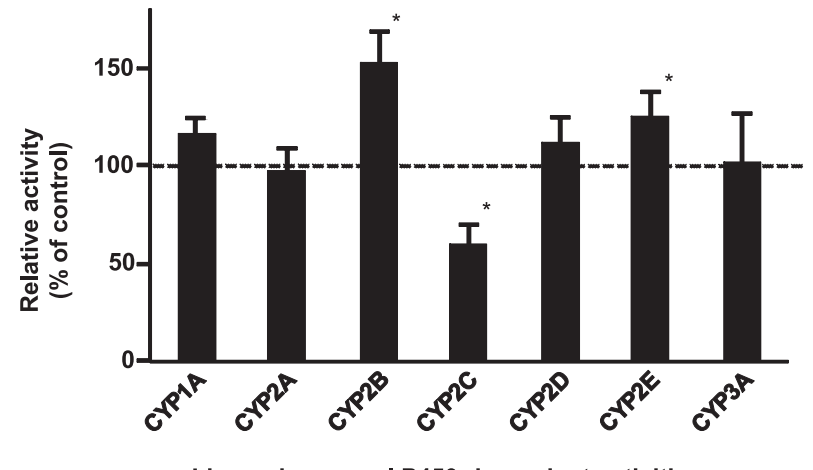

Liver microsomal P450-dependent activities

Fig. 5. Liver Microsomal P450-dependent Activities after 1,4dioxane i.p. Treatment

Control activities were taken from liver microsomes from untreated rats. Data columns with bars show mean \pm S.D. $(n=4)$. Significant differences compared with the control activities: ${ }^{*} p<0.05$. The i.p. treatment was conducted under the typical $\mathrm{P} 450$ induction by i.p. treatment with $\beta$-naphthoflavone, phenobarbital, and dexamethazone. $^{13)}$

Table 3. In Vitro Intrinsic Clearance of 1,4-dioxane Determined Using Liver Microsomes

\begin{tabular}{lcc}
\hline \hline Enzyme source & $\begin{array}{l}\text { Clearance, } \\
\mu 1 / \text { min per }\end{array}$ & $1 / h^{a)}$ \\
& mg protein & \\
\hline Rats, untreated $^{b)}$ & $45 \pm 13$ & 0.805 \\
Rats treated with 1,4-dioxane $^{b)}$ & $20 \pm 9$ & 0.313 \\
Pooled human livers $^{\text {Pat }}$ & 6.4 & 22.9 \\
\hline
\end{tabular}

1,4-Dioxane $(50 \mu \mathrm{M})$ was incubated with rat or human liver microsomes in the presence of an NADPH-generating system. The elimination rates of 1,4-dioxane were determined by GC/MS. a) Estimated clearance values were extrapolated using the following values: $40 \mathrm{mg}$ liver microsomal protein per $1 \mathrm{~g}$ liver and $10 \mathrm{~g}$ liver weight per $0.25 \mathrm{~kg}$ of rat bw or $1.5 \mathrm{~kg}$ liver per $70 \mathrm{~kg}$ of human bw. b) Mean \pm S.D. $(n=4)$ values using liver microsomes from individual rats i.p. pretreated with 1,4-dioxane $(500 \mathrm{mg} / \mathrm{kg})$ daily for 3 days or from untreated controls.

(7740 mg.h/l), giving $0.290 \mathrm{ml} / \mathrm{h}$. Primary hepatic clearance values of 1,4-dioxane were obtained by subtracting $\mathrm{CL}_{r}$ from the total clearance. Values of the plasma unbound fraction $\left(f_{u, p}\right)$ of 1,4-dioxane were calculated to be 0.806 by in silico estimation with SimCYP (Table 1).

$\mathrm{P} 450$ induction in rat liver microsomes was investigated after intraperitoneal treatment with 1,4dioxane for 3 days (Fig. 5). Judging from typical P450-dependent drug oxidation activities, P450 induction or suppression by repeated treatments with 1,4-dioxane seemed to be within 50-150\%, although CYP2B- and CYP2E-mediated activities were significantly induced and CYP2C-dependent activity was significantly decreased (Fig. 5). Consequently, final parameters such as hepatic intrin-

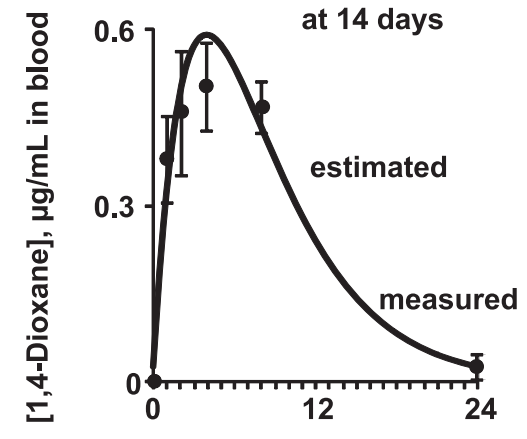

Time after administration (h)

Fig. 6. Measured and Estimated 1,4-dioxane Blood Concentrations in Rats after Daily Oral Administration $(500 \mathrm{mg} / \mathrm{kg})$ for 14 days

Data points with bars represent experimental mean \pm S.D. $(n=$ 5) as shown in Fig. 4A. The curve shows concentrations estimated by PBPK modeling.

sic clearance $\left(C L_{h, \text { int }}\right)$, volume of systemic circulation $\left(V_{1}\right)$, and absorption rate constant $\left(k_{a}\right)$ for the rat PBPK model were recalculated from the primary values by the user model in WinNonlin software to give $0.0244 \mathrm{l} / \mathrm{h}, 0.0810 \mathrm{l}$, and $0.280 \mathrm{~h}^{-1}$ and are shown in Table 1. By running the rat PBPK modeling system shown in Fig. 3, the blood concentration curves of 1,4-dioxane were estimated after repeated oral administration with $125 \mathrm{mg}$ of 1,4dioxane to a rat $(250 \mathrm{~g} \mathrm{bw})$; the curve is shown in Fig. 6. The estimated in silico concentration curve of 1,4-dioxane is shown with the in vivo experimental data points.Minimal accumulation was found by the present rat PBPK model $24 \mathrm{hr}$ after daily treatment with 1,4-dioxane in rats.

\section{Human PBPK Model Supported by In Vitro Hep- atic Clearance Experiments}

Hepatic clearance of 1,4-dioxane in vitro was determined in pooled human liver microsomes and compared with data from liver microsomes from rats pretreated with 1,4-dioxane and from untreated controls (Table 3). Hepatic clearance of 1,4-dioxane in human liver microsomes was calculated to be $6.4 \mu \mathrm{l} / \mathrm{min}$ per mg protein; this was apparently lower than the values for rat livers. Subsequently, the hepatic intrinsic clearance of 1,4-dioxane in humans was found be $22.9 \mathrm{l} / \mathrm{h}$ in an in vitro study using the biological coefficients already established. The intrinsic clearance values of 1,4-dioxane based on rat in vivo (Table 1) and rat in vitro (Table 3 ) experiments were different; this ratio $(0.0244 / 0.313)$ was used as the compensating factor for estimating in vivo hepatic intrinsic clearance in humans. Finally, 

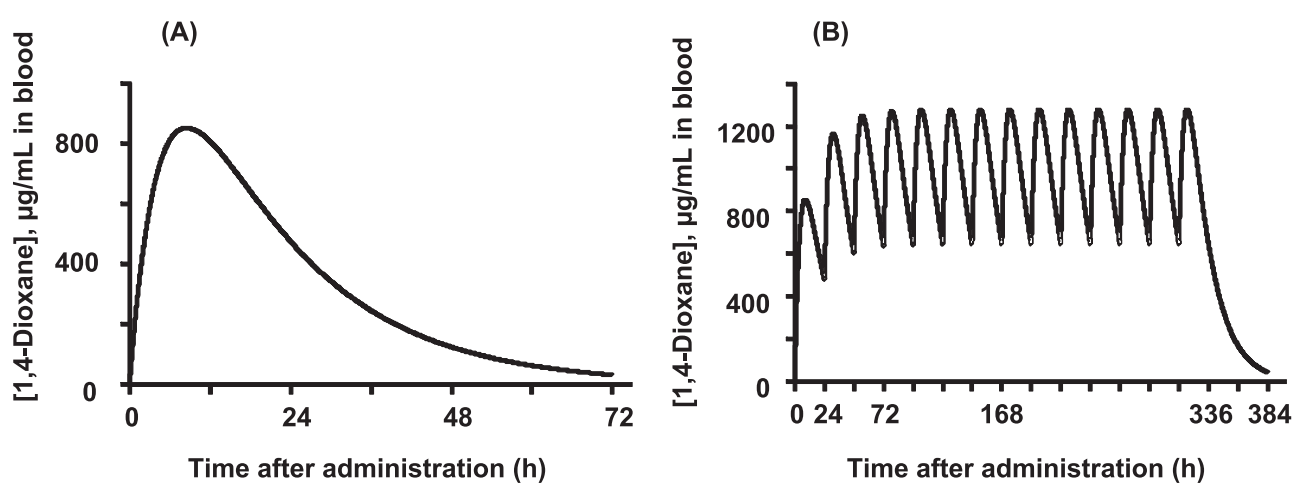

Fig. 7. 1,4-Dioxane Concentrations Hypothetically Modeled in Humans after Single (A) or Multiple (B) Oral Administrations $(500 \mathrm{mg} / \mathrm{kg}$ per day) Estimated Using the PBPK Model Little accumulation was observed for multiple doses.

a value of $1.76 \mathrm{l} / \mathrm{h}$ for the 1,4 -dioxane hepatic intrinsic clearance $\left(C L_{h, \text { int }}\right)$ was adopted to represent the in vivo status in the final human PBPK model, the parameters of which are shown in Table 2.

Figure 6 indicates the estimated human blood concentrations after modeling single and repeated oral administration of 1,4-dioxane $(500 \mathrm{mg} / \mathrm{kg})$. The apparent maximum concentrations of 1,4dioxane were estimated to be approximately $800 \mu \mathrm{g} /$ $\mathrm{ml}$ after a single treatment (Fig. 7A). When daily administration of 1,4-dioxane for 14 days was modeled, accumulation was found by the present human PBPK model (Fig. 7B).

\section{DISCUSSION}

It is generally accepted that PBPK modeling could be of use for understanding the relationship between chemical exposure and concentrations in body fluids (Fig. 2). However, the multiple compartments and many complicated equations found in traditional PBPK modeling cause severe difficulties when applying the model. Simple and reliable methods have not yet been established, but such models are needed to explore the biological significance of a wide range of chemicals. The present study defined a simplified PBPK model for 1,4dioxane in humans (Fig. 3); the model was based on physiological parameters derived from the literature, coefficients derived in silico, metabolic parameters determined in vitro using relevant liver microsomes, and in vivo experiment-supported PBPK modeling in rats. 1,4-Dioxane metabolic clearance is believed to be generally dependent on P450s, but there is no information regarding $\mathrm{P} 450$ isoforms in the 1,4-dioxane metabolism in rats or humans and its $\mathrm{P} 450$ induction/inhibition so far. ${ }^{7,8)} \mathrm{P} 450$ induction or suppression by repeated treatments with 1,4dioxane seemed to be limited in the present study, in spite of some increased or deceased marker oxidation activities (Fig. 5). Judging from effects of 1,4-dioxane on its some suppressed metabolic clearance in rat liver microsomes (Table 3), metabolic clearance of 1,4-dioxane may be mediated partly by CYP2C enzymes in male rats. The developed PBPK model for 1,4-dioxane in rats simply consisted of three compartments, including the gut as the chemical absorption compartment, the liver as the metabolizing compartment, and the general circulation as the central compartment for 1,4-dioxane (Fig. 3). Because of the simplicity of our adopted PBPK model, a reliable human hepatic clearance value and a complete set of human PBPK parameters could be estimated from limited experiments, namely oral dose administration in rats (Fig. 4) and in vitro clearance experiments with rat and human liver microsomes (Table 3 ) in the present study.

A critical review of the potential carcinogenicity of the weak genotoxicant 1,4-dioxane has indicated that the relevance of nasal cavity tumors to human exposure via the oral route is questionable. ${ }^{9)}$ A six-compartment PBPK model for 1,4-dioxane in humans has been reported. ${ }^{8)}$ Although oral administration of chemicals is a key route of exposure based on toxicology testing, ${ }^{10)}$ chemical concentrations absorbed from other routes, such as inhalation, could be handled in a similar PBPK modeling system. There are limited human data that can be used to validate the human model. Sweeney et al. ${ }^{8)}$ have reported a prediction of human subjects data for $50 \mathrm{ppm}$ inhalation for $6 \mathrm{hr}$ exposure to 1,4-dioxane. In that report, ${ }^{8)}$ plasma levels of 1,4-dioxane modeled in humans show some accumulation tendency 
similar to our predicted values after a single administration of 1,4-dioxane, as shown in Fig. 7A. In this context, some validation of the human PBPK model is needed in future study, based on the good agreement of measured and estimated blood concentrations in the rat PBPK model in this study.

Human biomonitoring is important for many aspects of environmental health. ${ }^{3,20)}$ Evaluation of a previously developed rat model was performed by comparing the blood concentrations predicted by PBPK modeling in silico and experimental biokinetic values from plasma and urine obtained from rats in vivo after repeated oral treatment with 1,4dioxane at an NOAEL. To overcome the species differences in animals and humans, the traditional parallelogram technique used in systems biology, ${ }^{21)}$ namely the concept of animal-human-in vitro-in vivo parallelogram in the risk assessment system ${ }^{22,23)}$, was adapted for this study to estimate the value of in vivo human hepatic clearance from in vitro data (Table 3). In summary, the present study indicates that simplified PBPK modeling for 1,4dioxane is useful for a forward dosimetry approach in rats and humans to estimate blood concentrations of 1,4-dioxane and other related 1,4-dioxane-like compounds from low chemical doses such as those at the NOAEL.

Acknowledgements This work was supported in part by JCIA's LRI program and by a Grant-in-Aid for High Technology Research Centre Project (198) from the Ministry of Education, Culture, Sports, Science and Technology of Japan.

Appendix A The liver-plasma concentration ratio $\left(K_{p, H}\right)$ was calculated from Eq. (A1): ${ }^{24)}$

$$
K_{p, h}=\frac{P \times 0.02289+0.72621}{P \times 0.00396+0.960581} \times \frac{f_{u, p}}{f_{u, h}}
$$

where $P$ is the water-octanol partition ratio and was estimated from the computer-calculated $\log P$ as neutral $(c \log P)$ :

$$
P=10^{\log P}
$$

$f_{u, h}$ is the hepatic unbound fraction for a specific binding on albumin, globulins, and lipoproteins. The tissue interstitial fluid-to-plasma concentration ratios of albumin, globulins, and lipoproteins were assumed to be 0.5 :

$$
\frac{f_{u, p}}{f_{u, h}}=0.5 \times\left(f_{u, p}+1\right)
$$

Appendix B The initial parameter values of $C L_{h \text {,int }}^{\prime}$ and $\mathrm{V}_{1}^{\prime}$ used to execute the fitting calculation of the PBPK model with WinNonlin software were derived from the following equations.

Hepatic clearance $\left(C L_{h}\right)$ was estimated from Eq. (B1), which was derived from Eq. (B2):

$$
\begin{aligned}
& C L_{h}=\frac{\text { Dose } \times Q_{h}-A U C \times C L_{r} \times Q_{h}}{A U C \times Q_{h}+\text { Dose }} \\
& \frac{C L_{\mathrm{tot}}}{F}=\frac{C L_{h}+C L_{r}}{1-\frac{C L_{h}}{Q_{h}}}=\frac{\text { Dose }}{A U C}
\end{aligned}
$$

where $A U C$ is the area under the curve.

The bioavailability $(F)$, fraction absorbed $\left(F_{a}\right)$, and intestinal availability $\left(F_{g}\right)$ are related as:

$$
F=F_{a} \times F_{g} \times F_{h}
$$

where $F_{h}$ is fraction unmetabolized in the liver.

In this study, we assume $F_{a} F_{g}=1.0$; then, the bioavailability was calculated from Eq. (B4) (the prime represents the value under the assumption of $\left.F_{a} F_{g}=1.0\right)$.

$$
F^{\prime}=F_{a} F_{g} \times F_{h}=F_{h}=1-\frac{C L_{h}}{Q_{h}}
$$

The initial value of $V_{1}$ was estimated from Eq. (B5) using the fitted calculation results of the one-compartment model $\left(V_{d} / F\right)$ and the $F^{\prime}$ value from Eq. (B4):

$$
V_{1}^{\prime}=\left(V_{d} / F\right) \times F^{\prime}
$$

The initial value of hepatic intrinsic clearance $\left(C L_{h, \text { int }}\right)$ was estimated from Eq. (B6), where $C L_{h}$ was evaluated from Eqs. (B7) and (B8):

$$
\begin{aligned}
& C L_{h, \text { int }}^{\prime}=\frac{R_{b}}{f_{u, p}} \times \frac{Q_{h} \times C L_{h}}{Q_{h}-C L_{h}} \\
& C L_{\text {tot }}=\left(V_{d} / F\right) \times k_{e l} \times F^{\prime} \\
& C L_{h}=C L_{\text {tot }}-C L_{r}
\end{aligned}
$$

Values of adjusted distribution volume $\left(V_{d} / F\right)$ and elimination constant $\left(k_{e l}\right)$ were calculated from the fitting calculation of the one-compartment model, and Eq. (B7) was derived from Eq. (B9):

$$
\frac{C L_{\mathrm{tot}}}{F}=\left(V_{d} / F\right) \times k_{e l}
$$

The initial value of $k_{a}$ for the fitting calculation was used as the primary results of WinNonlin with the one-compartment model.

Appendix $\mathbf{C}$ The parameter values of $C L_{r}, k_{a}$, and $V_{1}$ in the human PBPK model were estimated 
using a scale-up strategy from rats to humans as follows. Human renal clearance $C L_{r, \text { human }}$ was estimated from Eq. (C1), which was derived from Eq. $(\mathrm{C} 2)$, where $B W_{\text {rat }}=0.25 \mathrm{~kg}$ and $B W_{\text {human }}=$ $70 \mathrm{~kg}$ :

$$
\begin{aligned}
& C L_{r, \text { human }}=\frac{C L_{r, \text { rat }}}{B W_{r a t}^{2 / 3}} \times B W_{\text {human }}^{2 / 3} \\
& C L_{r}=a \times B W^{2 / 3}
\end{aligned}
$$

The human systemic circulation volume $\left(V_{1, \text { human }}\right)$ was estimated from Eqs. (C3) and (C4), where $V_{h, \text { rat }}, V_{h, \text { human }}, V_{b, \text { rat }}$, and $V_{b, \text { human }}$ were 0.00851 , $1.51,0.0161$, and 4.91 , respectively:

$$
\begin{aligned}
V_{1, \text { human }}= & V_{d, \text { human }}-V_{h, \text { human }} \times \frac{K_{p, h} \times F_{h}}{R_{b}} \\
V_{d, \text { human }}= & V_{b, \text { human }}+\left(V_{d, \text { rat }}-V_{b, \text { rat }}\right) \times \frac{R_{b, \text { rat }}}{f_{u, p, \text { rat }}} \\
& \times \frac{f_{u, \text { }, \text { human }}}{R_{b, \text { human }}}
\end{aligned}
$$

where physicochemical parameters such as $K_{p, h}, R_{b}$, and $f_{u, p}$ were assumed to be consistent between rats and humans. The derivation of $K_{p, h}$ is shown in Appendix A.

The human absorption rate constant $\left(k_{a}\right)$ was estimated from Eq. (C5). ${ }^{25)}$

$$
k_{a, \text { human }}=0.744 \times k_{a, r a t}
$$

\section{REFERENCES}

1) Hayes, K. R. and Bradfield, C. A. (2005) Advances in toxicogenomics. Chem. Res. Toxicol., 18, 403414.

2) Edwards, S. W. and Preston, R. J. (2008) Systems biology and mode of action based risk assessment. Toxicol Sci., 106, 312-318.

3) Collins, F. S., Gray, G. M. and Bucher, J. R. (2008) Toxicology. Transforming environmental health protection. Science, 319, 906-907.

4) Wild, C. P. (2005) Complementing the genome with an "exposome": the outstanding challenge of environmental exposure measurement in molecular epidemiology. Cancer Epidemiol. Biomarkers Prev., 14, 1847-1850.

5) Clewell, H. J., Gentry, P. R., Covington, T. R., Sarangapani, R. and Teeguarden, J. G. (2004) Evaluation of the potential impact of age- and gender-specific pharmacokinetic differences on tissue dosimetry. Toxicol. Sci., 79, 381-393.
6) Guengerich, F. P. (2008) Cytochrome P450 and chemical toxicology. Chem. Res. Toxicol., 21, 7083.

7) Krishnan, K. and Johanson, G. (2005) Physiologically-based pharmacokinetic and toxicokinetic models in cancer risk assessment. J. Environ. Sci. Health C Environ. Carcinog. Ecotoxicol. Rev., 23, $31-53$.

8) Sweeney, L. M., Thrall, K. D., Poet, T. S., Corley, R. A., Weber, T. J., Locey, B. J., Clarkson, J., Sager, S. and Gargas, M. L. (2008) Physiologically based pharmacokinetic modeling of 1,4-dioxane in rats, mice, and humans. Toxicol. Sci., 101, 32-50.

9) Stickney, J. A., Sager, S. L., Clarkson, J. R., Smith, L. A., Locey, B. J., Bock, M. J., Hartung, R. and Olp, S. F. (2003) An updated evaluation of the carcinogenic potential of 1,4-dioxane. Regul. Toxicol Pharmacol., 38, 183-195.

10) Kano, H., Umeda, Y., Saito, M., Senoh, H., Ohbayashi, H., Aiso, S., Yamazaki, K., Nagano, K. and Fukushima, S. (2008) Thirteen-week oral toxicity of 1,4-dioxane in rats and mice. J. Toxicol. Sci., 33, 141-153.

11) Leung, H. W. and Paustenbach, D. J. (1990) Cancer risk assessment for dioxane based upon a physiologically-based pharmacokinetic approach. Toxicol. Lett., 51, 147-162.

12) Reitz, R. H., McCroskey, P. S., Park, C. N., Andersen, M. E. and Gargas, M. L. (1990) Development of a physiologically based pharmacokinetic model for risk assessment with 1,4-dioxane. Toxicol. Appl. Pharmacol., 105, 37-54.

13) Yamazaki, H., Shimizu, M., Nagashima, T., Minoshima, M. and Murayama, N. (2006) Rat cytochrome P450 2C11 in liver microsomes involved in oxidation of anesthetic agent propofol and deactivated by prior treatment with propofol. Drug Metab. Dispos., 34, 1803-1905.

14) Omura, T. and Sato, R. (1964) The carbon monoxide-binding pigment of liver microsomes. I. Evidence for its hemoprotein nature. J. Biol. Chem., 239, 2370-2378.

15) Murayama, N., Kaneko, N., Horiuchi, K., Ohyama, K., Shimizu, M., Ito, K. and Yamazaki, H. (2009) Cytochrome P450-dependent drug oxidation activity of liver microsomes from Microminipigs, a possible new animal model for humans in non-clinical studies. Drug Metab. Pharmacokinet., 24, 404-408.

16) Yamazaki, H., Mimura, M., Sugahara, C. and Shimada, T. (1994) Catalytic roles of rat and human cytochrome P450 2A enzymes in testosterone $7 \alpha$ and coumarin 7-hydroxylations. Biochem. Pharmacol., 48, 1524-1527. 
17) Yamazaki, H., Nakamura, M., Komatsu, T., Ohyama, K., Hatanaka, N., Asahi, S., Shimada, N., Guengerich, F. P., Shimada, T., Nakajima, M. and Yokoi, T. (2002) Roles of NADPH-P450 reductase and apo- and holo-cytochrome $b_{5}$ on xenobiotic oxidations catalyzed by 12 recombinant human cytochrome P450s expressed in membranes of Escherichia coli. Protein Expr. Purif., 24, 329-337.

18) Kato, M., Shitara, Y., Sato, H., Yoshisue, K., Hirano, M., Ikeda, T. and Sugiyama, Y. (2008) The quantitative prediction of CYP-mediated drug interaction by physiologically based pharmacokinetic modeling. Pharm. Res., 25, 1891-1901.

19) Emoto, C., Murayama, N., Rostami-Hodjegan, A. and Yamazaki, H. (2009) Utilization of estimated physicochemical properties as an integrated part of predicting hepatic clearance in the early drugdiscovery stage: Impact of plasma and microsomal binding. Xenobiotica, 39, 227-235.

20) National Research Council of the National Academies (2007) Toxicity Testing in the 21st Century: A Vision and a Strategy, The National Academies Press, Washington, D.C.

21) Hays, S. M., Becker, R. A., Leung, H. W., Aylward, L. L. and Pyatt, D. W. (2007) Biomonitoring equivalents: a screening approach for interpreting biomon- itoring results from a public health risk perspective. Regul. Toxicol. Pharmacol., 47, 96-109.

22) Takano, R., Murayama, N., Horiuchi, K., Kitajima, M., Kumamoto, M., Shono, F. and Yamazaki, H. Blood concentrations of acrylonitrile in humans after oral administration extrapolated from in vivo rat pharmacokinetics, in vitro human metabolism, and physiologically based pharmacokinetic modeling. Regul. Toxicol. Pharmacol., in press.

23) Yamazaki, H., Takano, R., Horiuchi, K., Shimizu, M., Murayama, N., Kitajima, M. and Shono, F. (2010) Human blood concentrations of dichlorodiphenyltrichloroethane (DDT) extrapolated from metabolism in rats and humans and physiologically based pharmacokinetic modeling. J. Health Sci., 56, 566-575.

24) Poulin, P. and Theil, F. P. (2002) Prediction of pharmacokinetics prior to in vivo studies. 1. Mechanismbased prediction of volume of distribution. $J$. Pharm. Sci., 91, 129-156.

25) Amidon, G. L., Sinko, P. J. and Fleisher, D. (1988) Estimating human oral fraction dose absorbed: a correlation using rat intestinal membrane permeability for passive and carrier-mediated compounds. Pharm. Res., 5, 651-654. 